\title{
EL REPERTORIO LÉXICO-SEMÁNTICO DE LA PRENSA AMARILLA EN LIMA METROPOLITANA
}

\author{
Lic. Edward Faustino Loayza Maturrano \\ Profesor del Departamento de Ciencias Humanas de la \\ Universidad Nacional Agraria La Molina \\ edwloma@lamolina.edu.pe
}

\begin{abstract}
Resumen
Esta investigación trata del análisis léxico-semántico del lenguaje de la prensa amarilla escrita en Lima Metropolitana. Su propósito es poner en práctica un análisis lexical y semántico de la fraseología que se emplea en estos medios de comunicación masiva, específicamente en la prensa escrita amarillista en la ciudad de Lima. A su vez, este estudio permite conocer la lengua en uso en el Perú, puesto que los diarios son la síntesis de la idiosincrasia limeña.

Es una investigación descriptiva dentro de la teoría de la lingüística estructural, en la que se estudian qué factores influyen en la determinación del repertorio de la prensa amarillista, cuáles son sus características; así como los mecanismos de innovación léxica subestándar más frecuentes. Al propio tiempo, se desarrolla el análisis lexicológico y semántico respectivo, empleando las variables propias del nivel lingüístico aplicadas a las diferentes variaciones léxico-semánticas del lenguaje de la prensa amarilla.
\end{abstract}

Palabras Claves: Prensa amarilla, léxico, semántico, subestándar, lengua, innovación léxica.

\begin{abstract}
This research deals with the lexical-semantic analysis of the language of tabloid newspapers written in Metropolitan Lima. Its purpose is to implement a lexical and semantic analysis of phraseology that is used in the mass media, specifically in the yellow print press in the city.

In turn, this study allows to know the language used in Peru, because newspapers are the synthesis of the idiosyncrasies of Lima. A descriptive research in the theory of structural linguistics, examines what factors influence the determination of the register of the yellow print press, what are its characteristics, as well as the mechanisms of lexical innovation frequently substandard. At the same time develops issue about lexical and semantic analysis, using the variables specific to the linguistic level applied to different variations of the lexical-semantic language of of the yellow print press.
\end{abstract}

Key words: Yellow print press, lexical, semantic, sub-standard language, lexical innovation. 


\section{Introducción}

El análisis del repertorio léxico-semántico de la prensa amarilla en Lima se ha de efectuado considerando el ámbito estructural (morfológico) de la palabra (morfemas derivativos: prefijos, infijos y sufijos) y los morfemas léxicos o lexemas, este análisis se completó identificando el tipo de palabra según su formación (palabra primitiva, derivada, compuesta y parasintética). De igual modo, se determinó la clase de palabra a la que pertenece, esto es, sustantivo, adjetivo, verbo, adverbio, preposición, conjunción, artículo, pronombre.

Este trabajo plantea el siguiente problema principal de investigación:

¿Cuáles son las características del repertorio léxico-semántico de la prensa amarilla en la ciudad de Lima en las condiciones de la realidad actual?

La solución de este problema se logró dando respuesta previa a los siguientes subproblemas, derivados de él:

¿Qué factores influyen en el establecimiento del repertorio léxico-semántico de la prensa amarilla en la ciudad de Lima en las condiciones de la realidad actual?; ¿Cuáles son las características léxicas del repertorio lingüístico de la prensa amarilla en la ciudad de Lima en las condiciones de la realidad actual?; ¿Cuáles son las características semánticas del repertorio lingüístico de la prensa amarilla en la ciudad de Lima en las condiciones de la realidad actual?; ¿De qué manera un análisis léxico-semántico permite determinar con adecuación y coherencia la situación lingüística de la prensa amarilla en la ciudad de Lima en las condiciones de la realidad actual?

Sobre la base del problema científico planteado se definieron los siguientes objetivos para la investigación:

Determinar las características del repertorio léxico-semántico de la prensa amarilla en la ciudad de Lima en las condiciones de la realidad actual.

De este objetivo general se desprendieron los siguientes objetivos específicos:

Identificar los factores que influyen en el establecimiento del repertorio léxicosemántico de la prensa amarilla en la ciudad de Lima en las condiciones de la realidad actual. Determinar las características léxicas del repertorio lingüístico de la prensa amarilla en la ciudad de Lima en las condiciones de la realidad actual. Determinar las características semánticas del repertorio léxico de la prensa amarilla en la ciudad de Lima en las condiciones de la realidad actual. Desarrollar un análisis léxico-semántico que permita determinar con adecuación y coherencia la situación lingüística de la prensa amarilla en la ciudad de Lima en las condiciones de la realidad actual.

La hipótesis principal que se formuló fue:

El repertorio léxico-semántico de la prensa amarilla en la ciudad de Lima en las condiciones de la realidad actual pertenece a la jerga marginal del sociolecto delincuencial.

Las hipótesis específicas que se desprenden de la anterior fueron: 
El repertorio léxico-semántico de la prensa amarilla en la ciudad de Lima en las condiciones de la realidad actual infringe la normativa castellana, empleando como estrategia de formación léxica el apócope, transposición morfemática, y el trastrocamiento y cambio fonémico. El repertorio léxico-semántico de la prensa amarilla en la ciudad de Lima en las condiciones de la realidad actual, incluye también al sociolecto juvenil.

La variable principal de la presente investigación fue: El análisis léxico-semántico de la prensa amarilla. De esta variable se desprende las siguientes subvariables e indicadores:

\section{a. Análisis léxico}

\section{a.1) Análisis estructural}

Morfema léxico o lexema, Morfemas derivativos, Morfemas flexivos, Morfema de género, Morfema de número, Morfema de tiempo (en el caso de verbos), Morfema de persona (en el caso de verbos), Morfema de modo (en el caso de verbos).

\section{a.2. Fenómeno léxico}

Préstamos léxicos, Innovaciones léxicas por reestructuración.

\section{b. Análisis semántico}

Semas comunes, Semas distintos, El semema de la palabra (significado denotativo), El virtuema de la palabra (significado connotativo), El(los) clasema(s), La hiperonimia / hiponimia, La polisemia, Sinonimia, Antonimia.
Los antecedentes del estudio está conformado por la investigación de Juana Elizabeth Jofré Burgos (2004) en su tesis "Las innovaciones léxicas como recurso expresivo en la prensa escrita: Caso revista El Sábado de El Mercurio” presentada en la Universidad Austral de Chile aborda la problemática de la incidencia de la prensa escrita en la incorporación de las innovaciones léxicas del área médica en la lengua castellana circunscrita a Chile.

En segundo lugar, Juan Gargurevich Regal (2002) en su publicación "La prensa sensacionalista del Perú” estudia al periodismo amarillo o chicha desde una óptica sociológica más no desde una óptica lingüística, la cual es el motivo del presente estudio.

Fedor Larco Degregori (2000) en su "Diccionario de la jeringa peruana" muestra el repertorio lingüístico de la jerga peruana en los últimos años. A partir de este estudio podemos conocer el bagaje subestándar del léxico-semántico peruano.

El sustento teórico del estudio está dado, en primer término por enunciar que el fenómeno de la llamada "prensa amarilla" no es un elemento aislado y totalmente novedoso en la historia de la prensa peruana. Ciertamente tiene rasgos que lo diferencian notablemente de la prensa política y seria, pero comparte una historia común con una serie de proyectos de diarios sensacionalistas que surgieron sobre todo en la segunda mitad del siglo pasado, paralelamente con el crecimiento de la población urbana. 
Se suele decir que para cada medio hay un público; sin embargo, también es cierto que para cada forma de ver el mundo puede surgir un medio. Estamos hablando, por lo tanto, de un doble proceso en el que María Mata, acuciosa investigadora argentina, encuentra acertadamente que los medios constituyen a los públicos y a su vez los sujetos re-elaboran las ofertas y pueden diferenciarse de otros grupos en la interpretación de las mismas. (Cf. Mata, 2000: 19)

En ese sentido, es necesario comprender que los públicos no son solo un producto de la oferta actual de los medios (enfoque sincrónico) sino que han constituido sus gustos, intereses y motivaciones a lo largo del tiempo en complejos y continuos mecanismos de negociación entre lo que viven cotidianamente y lo que encuentran en los diarios, la TV y en la radio (enfoque diacrónico). Las percepciones de los públicos se conforman a través del consumo directo (como lectores habituales o lectores casuales) o a través de las relaciones personales que establecen con padres, amigos, instituciones. Los jóvenes, por ejemplo, no necesitan consumir MTV para pensar el mundo de modo "cool"; esto también se transmite y recrea a través de los compañeros del colegio y del barrio.

Lo mismo ocurre con la prensa sensacionalista. Existe un tradicional uso de la jerga, de la burla, de la narrativa exagerada, "amarilla", la cual es tomada por los medios populares. En este artículo examinaremos brevemente el derrotero de dicha prensa, sin la intención de hacer un recuento histórico, sino solo de rescatar sus esfuerzos y el interés de ciertos públicos por las noticias que se sitúan al margen de los parámetros tradicionales de la prensa política y seria.

Paralelamente, nos interesa esbozar la manera como estos medios satisfacían las demandas de protagonismo y representación de las plebes urbanas carentes de rostro público y presencia social. Esto nos sitúa en un paradigma diferente al análisis del periodismo como un conjunto de técnicas y metodologías de la veracidad y de la ética. En su lugar, nos conduce a un terreno más complejo pero algo fangoso, según el cual la forma en que se produce, brinda y consume información no es una forma pura, exenta de la historia y de la coyuntura sino, como Langer sugiere, es pensar "las noticias como discurso cultural”. (Langer, 2000: 213)

Así, los medios sensacionalistas no surgen de la nada. Por el contrario, continúan una tendencia iniciada por el periodismo en los años cincuenta, de la cual podemos identificar por lo menos cuatro etapas, las que nos permiten comprender que las complejas relaciones entre morbo, crónica roja y vedettismo que los diarios tejen con los públicos se constituyen en el tiempo.

Siguiendo a Wilbur Schramm (1967), quien identificó tres procesos sociales en los que se insertan los medios de comunicación masivos, los cuales son pasibles de extrapolar al análisis de la prensa amarilla.

Estos tres procesos sociales son:

1. Centran la atención del público sobre determinados asuntos.

2. Influyen limitadamente en la toma de decisiones (mediatizados por la acción de los líderes de opinión). 


\section{Actúan como entes pedagógicos.}

La relevancia de este último punto también la expresa G. Rodríguez (1994: 30) es el carácter masivo de la prensa amarilla lo que lo transforma en una verdadera escuela socioeducativa y muchas de las modas sociales o lingüísticas provienen de los medios nacionales de información. Y como el cambio en la lengua es la difusión o generalización de una innovación en una determinada comunidad de hablantes, es en este contexto donde realiza su contribución la prensa escrita.

Carlos Lomas (en M. Vaquero, 1999: 22), manifiesta que el lenguaje de la prensa ha de ser objeto de uso, (y abuso) en las hablas de la lengua. En la prensa encontramos un caudal casi infinito de diferentes tipos de textos (expositivos, argumentativos, descriptivos, narrativos, conversacionales, literarios...), con los que alude a diversos aspectos de la realidad cotidiana y que actúan no sólo como un reflejo de los distintos géneros textuales, sino también de las diferentes funciones del lenguaje periodístico (informar, narrar, describir, argumentar, opinar...). Porque, aunque a la prensa, como a la radio, o a la televisión se le atribuya esencialmente la función de informar, un análisis semiológico del lenguaje de los medios de comunicación de masas confirma que con él no sólo se informa sobre el mundo (hacer saber), sino también se persuade (hacer creer), se emociona (hacer sentir) y se manipula a las personas (hacer parecer verdad y hacer hacer).

En segundo término es de vital importancia determinar los principales factores que influyen en el establecimiento del repertorio léxico-semántico de la prensa amarilla, entre los cuales tenemos:

\section{a. Los factores socioculturales}

El lenguaje es el único tipo de conducta social cuya función primaria es la comunicación, y toda acción comunicativa implica el uso de signos (lo cual estudia la semiótica). Entendiendo así, se transforma en una de las mayores riquezas de una persona y de un pueblo, dado que es el principal medio de comunicación entre las mismas. Por ende, todo lo que se produce en relación con el lenguaje sucede para ser comunicado en el intercambio social. La misma palabra comunicar conlleva la idea de común, comunidad, por lo tanto, factores sociales de distinta índole como el tamaño del grupo humano, las características individuales y los efectos de la interacción entre estos grupos, forman parte del complejo mundo de la comunicación. Así la competencia comunicativa siempre está presente en un acto de comunicación efectiva con la finalidad que la transmisión de información se realice, y el hablante comunique sus significados e intenciones al oyente. De este modo, cada hablante estructura a través de su sistema lingüístico la realidad amorfa, reflejándose los modelos sociales y culturales en la estructura de la lengua, es decir, en el código lingüístico que da origen al lenguaje. (Malmberg, 1966: 62)

Entonces, todo análisis de la cultura y la vida social, bajo cualquiera de sus formas siempre cambiantes, es también y en gran medida un análisis del lenguaje humano, de las convenciones, del sistema lingüístico. 
Como se ha señalado, todo acto comunicativo es un proceso de interpretación de intenciones, cuyo objetivo es el intercambio de información que se transmite a través de la lengua organizada a través de dos tipos de conocimientos:

1. Explícito o gramatical: éste se elabora a partir de los conocimientos gramaticales, es el significado que se desprende de las palabras que forman el enunciado, es decir, las que el hablante-oyente ha adquirido durante la etapa de aprendizaje y que utiliza en su interacción social, permitiéndole expresar una cantidad ilimitada de pensamientos nuevos adaptados a situaciones nuevas.

2. Implícito o contextual: es el que se construye a partir de los conocimientos pragmáticos. Es el conocimiento adicional que permite al oyente interpretar adecuadamente las palabras del hablante, obtenidas de todas aquellas convenciones y circunstancias del entorno-compartido por los interlocutores en que se produce el enunciado- como el bagaje sociocultural en que están insertos. Un mismo enunciado se puede interpretar de distintas formas, pero dependiendo de este tipo de información se contextualiza el mensaje en una determinada situación comunicativa y en un entorno sociocultural específico, lo que permite restringir el conjunto de opciones interpretativas.

De tal manera, una planificación o sincronización entre los interlocutores, quienes estructuran sus mensajes para lograr sus ob- jetivos sociales, infiriendo para ello sus estados mentales en función del conocimiento previo de las características observables y de la situación en la que se encuentra.

Es decir, contextualizan la comunicación distinguiendo entre lo lingüístico y la situación de la experiencia vivida no lingüística. Se traduce como medios propiamente lingüísticos lo que es pertinente en la situación para constituir el mensaje. Por ejemplo, en mujer fatal y accidente fatal, mujer y accidente son el contexto. En el primer caso la interpretación es metafórica y en el segundo es literal. Igual cosa sucede con los enunciados: fiesta mortal y golpe mortal. Otro ejemplo sería: A Juan se le apagó la tele y Juan apagó la tele. El primer caso da cuenta de una situación y en el segundo de una acción.

Esta sensibilidad y capacidad de acomodar está en el corazón de la comunicación, coordina el ajuste del acto comunicativo como el eje central de todos los niveles del lenguaje, desde las palabras aisladas, a las conversaciones y narrativas.

Parece obvio que la comunicación no es posible si los interlocutores no están de acuerdo en una lengua común, en un vocabulario común y en una sintaxis ${ }^{1}$. El sistema lingüístico concreto que en cada ocasión emplean los hablantes viene dictado no sólo por consideraciones de mutua inteligibilidad, sino también en función de variables tales de las situaciones del habla como: con quién se encuentra el hablante; dónde tiene lugar la conversación; sobre qué versa; qué tipos de intercambios han precedido a la situación considerada ( $C f$. Gumperz 1981: 119). 
En general, la opción interpretativa que se escoge es la adecuada para que se produzca la comunicación, y el oyente interpreta los enunciados del hablante de acuerdo con la misma intención y la finalidad con la que éste los produce. Ocurre así, porque todo acto comunicativo está regulado por el Principio de Cooperación formulado por Grice (en Susana Luque 2000: 17), que postula que los enunciados que emiten los participantes en un acto comunicativo están siempre encaminados a que la comunicación sea efectiva.

El Principio de Cooperación lo formula en cuatro máximas:

1. Cantidad: haga su contribución tan informativa como sea necesario para el objetivo del intercambio comunicativo en el que se haya inmerso, y no haga su contribución más informativa de lo necesario.

2. Calidad: trate de que su contribución sea verdadera. No diga lo que cree que es falso, o no diga algo de lo cual carece de pruebas adecuadas.

3. Relación: trate de que sus contribuciones sean pertinentes.

4. Manera: sea claro, evite la oscuridad y la ambigüedad en la expresión. Sea breve y ordenado.

A la competencia comunicativa, se debe sumar por parte de los interlocutores el dominio del sistema lingüístico que está conformado por los niveles fonético, gramatical y léxico. Sin este último no se logrará concretar la comunicación.
Por nivel fonético se entiende el estudio del modo sistémico y reglado como los humanos combinan los sonidos para formar palabras. Se ocupa fundamentalmente de la descripción y la clasificación de los sonidos producidos en los actos de habla humanos (Cf. Gumperz, 1981: 23 -24).

El conocimiento lingüístico es el sistema de reglas interiorizado por los hablantes y que constituye su saber lingüístico, gracias al cual son capaces de pronunciar o de comprender un número infinito de oraciones inéditas. El sistema de reglas que gobiernan a una lengua en su conjunto, es a lo que los lingüistas dan el nombre de gramática. (Cf. Gumperz, 1981: 23 $-24)$.

En cuanto al nivel léxico se hará referencia a dos definiciones. La primera señala que léxico es el conjunto de las unidades significativas de una lengua dada, en un momento determinado de su historia (Mounin, 1979:114). La segunda, define léxico como el conjunto o sistema de palabras que componen una lengua $(C f$. Lewandowski, 2000:208). Es a este último nivel (léxico) al que se remitirá la presente investigación. Se estudiarán las palabras, considerando su nivel semántico, es decir, analizando su significado.

\section{b. Factores espaciales}

Los factores espaciales son aquellos elementos geográficos que influyen directa e indirectamente en algún tipo de variación lingüística. En este sentido, podemos nombrar algunos factores de influencia en la lengua, a saber: El clima, la naturaleza del suelo, la vegetación existente, la fau- 
na del lugar. Es decir, el ecosistema y los fenómenos meteorológicos son los factores geográficos de mayor influencia en la lengua.

A este tipo de variación lingüística se le denomina variación diatópica, ya que se diferencia en función del espacio e indica que todos los hablantes de una lengua proceden de una determinada zona geográfica. Esta variación lingüística es estudiada por la dialectología, rama de la sociolingüística.

\section{c. Factores temporales}

El tiempo es uno de los factores que influye en la variación lingüística. Esta variación por influencia temporal puede darse en un plano sincrónico y en un plano diacrónico. La proyección de la variación a través de estados sincrónicos, o de un momento histórico dado, se representan por las siguientes unidades: el dialecto, para la variedad diatópica; el sociolecto, en el caso de la variedad diastrática; y el registro, para la variación diafásica.

La variedad diacrónica es aquella que implica una diferencia en el tiempo y que es abordada por la historia de la lengua. En este plano, la lengua sufre cambios y no solo variaciones. Un cambio lingüístico supone el paso de un estado a otro. Por tanto, las simples aportaciones al cambio que dan los sujetos de forma individual o social sólo de hace cualitativo cuando implica una nueva lógica lingüística o un nivel de desarrollo distinto.

La lengua viene a ser un elemento vivo, es decir que se transforma, cambia de acuer- do a las necesidades de una comunidad lingüística. Por ello, es que las innovaciones y diferencias léxicas responden en términos generales a variaciones dialectales o dialectos de variedades funcionales o registros con que se identifica cada comunidad. Existen factores como los geográficos (dialectos), socioculturales (sociolectos) y generacionales o temporales que influyen en esta diferenciación, respondiendo al carácter dinámico de la lengua.

En este contexto se puede indicar que (Prieto, 1990), una situación de comunicación sobrepasa a las herramientas que se utilizan para comunicarse -colectiva o individualmente- ya que ella comprende las relaciones intrapersonales, grupales y sociales; las circunstancias económicas, políticas y culturales; el desarrollo de ciertas tecnologías, de ciertas formas de enfrentar y resolver los problemas de la naturaleza y la sociedad. Entonces, el lenguaje es el medio utilizado para la comunicación y es a través que se obtiene el conocimiento cultural de una comunidad específica.

En este mismo sentido, Albert Schulz (Saperas, 1987, cit. a Schulz; en Ruiz, 1998; en Mora, 1999) señala que el "lenguaje y la comunicación desempeñan un rol fundamental en la comprensión de la realidad social, ya que ésta sólo existe en función de que la comunicación -a través del lenguaje- permite disponer de un mecanismo de objetivación”.

Se comprende el entorno a través del lenguaje, lo que conlleva en sí mismo modificación, dinamismo, debido a que no todas las personas lo comprenden de la misma forma. Afirmación que realiza 
Akmajian (1995) de la siguiente forma, "ninguna lengua humana puede decirse que es fija, uniforme o invariable. Todas ellas muestran alguna variación interna en el sentido de que se transforman según su uso cotidiano por parte de cada uno de los hablantes. Se van diferenciando entre sí, ya sea, por la pronunciación de esas lenguas, la elección de las palabras del vocabulario y sus propios significados e incluso, por el empleo de determinadas construcciones sintácticas".

La adaptación del lenguaje al entorno en donde los interlocutores se comunican, se denomina variedad lingüística y es la que "permite que los hablantes mejoren su conocimiento de la realidad en que viven y se comuniquen mejor en ella" (Medina, 1994; en Luque, 2000). Por ende, se entiende que la variación dialectal responde -como se señaló en los párrafos anteriores- a factores socio-geográfico de los hablantes y a las distintas situaciones contextuales en la que se desenvuelve el acto comunicativo - variación funcional (Luque, 2000).

En cuarto orden, es necesario indicar los principales mecanismos de innovaciones lingüísticas:

a) Alteracióndeun modelotradicional: aquí existe una modificación en la base mediante la adición de elementos que complementan su significado o le asignan una función morfosintáctica, por ejemplo, unas pequeñas unidades que no tienen vida independiente, los afijos, que pueden agregarse al final de una base, al principio, o en medio de ella. b) Creación sistemática: invención de formas de acuerdo al sistema lingüístico, o sea, a la agregación prefijal, sufijal u otras. Ejemplos: chorear, chatear.

c) Préstamo de otra lengua: puede ser total o parcial $y$, con respecto a su modelo, puede implicar también alteración.

Dentro de estos tipos de innovaciones, es posible destacar la existencia de distintos procesos que influyen en la variación léxica:

\section{c.1) Uso de palabras antiguas,} adaptadas a una nueva función cambiándoles el significado;

c.2) Uso de metáforas en palabras preexistentes en la lengua, con nuevos contenidos para aumentar el significado de una palabra habitual;

c.3) La transformación de los nombres propios en sustantivos;

d) Los préstamos de palabras provenientes de otras lenguas o campos semánticos (de palabras inglesas, francesas o nativas; el uso de palabras propias de un área específica, por ejemplo, del campo de la biología). Dentro de los préstamos lingüísticos se pueden identificar los siguientes procesos de innovación:

- La homonimia que es recurrir a una misma palabra otorgándoles dos o más contenidos distintos (Kaposi en el ámbito de las enfermedades); 
- La metonimia que es designar un producto por su marca o lugar de origen de ésta (ejemplo: gillete);

- La antonomasia, que es generalizar las características propias de un personaje utilizando su nombre propio como común (ejemplo, toledismo).

- Calco lingüístico, que es la traducción de un préstamo;

- Los híbridos, que es la mezcla de una palabra espańola con una de otra lengua;

e) La composición de palabras, a través de la adición de elementos que complementan su significado o le asignan una función morfosintáctica. En esto se encuentra el uso de:

Prefijos (a-, anti-, des-, ultra-, auto-, etc.);

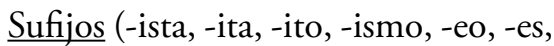
-ción, etc.);

Sintagmas fijos, suma de dos palabras independientes creando una nueva (como comecuras);

\section{f) La descomposición de palabras,} es decir, eliminando alguno de sus componentes originales. Aquí se pueden encontrar:

La elipsis, que es la eliminación de una gran parte de los elementos de conforman un concepto (por ejemplo Concertación, por Concertación de Partidos por la Democracia);

La aglutinación donde se funden los elementos de una frase compleja eliminando elementos intermedios (ejemplo, biosicosocial por biología, sicología y social);

Uso de siglas para reducir los nombres muy largos ( ejemplo: Servicio de Inteligencia Nacional, SIN). Estos elementos léxicos admiten derivaciones propias para darle distintos énfasis, sin que sea necesario volver a describir su sintagma original.

La reducción, eliminando partes de una palabra ya sean sufijos, prefijos, sílabas, etc. transformándose en un lenguaje más coloquial (ejemplo: depre por depresión);

La abreviación que es un procedimiento gráfico en que se dejan sólo algunos grafemas que evocan a la palabra para no escribirla completa, pero que en algunos casos ha pasado al lenguaje oral (ejemplo: depa por departamento).

g) La Derivación de palabras: en el español y especialmente en Lima, es muy productiva la derivación por sufijación diminutiva, con el fin de evitar expresiones que puedan resultar "duras" o violentas. Ejemplo, se prefiere teclito en vez de abuelo.

También es común el uso de diminutivo con carácter de atenuante. Ejemplo: espéreme un ratito, convídame un poquito, tiene un niñito cieguito. En todos los casos antes descritos, la derivación diminutiva al menos en Lima, tienen también un carácter de eufemismo.

Con el sufijo -ada se producen por derivación formas frecuentes como: se pegó una arrancada. 
Con el sufijo aumentativo -on se observa el conocido folclóricamente el conocido guatón Loyola, o el cabezón, etc. La estrecha relación entre la innovación por derivación y la creación sistemática es evidente.

\section{Materiales y métodos}

Los instrumentos que se ha creído pertinente utilizar en la investigación fueron: El análisis de documentos (diversos diarios amarillos de edición diaria en Lima Metropolitana).

El análisis del repertorio lexicológico de la prensa amarilla se efectuó considerando el ámbito estructural (morfológico) de la palabra. Esto significa que se analizaron sus unidades mínimas de significado incompleto, es decir, los morfemas. Entre los principales morfemas están los derivativos (prefijos, infijos y sufijos) y los morfemas léxicos o lexemas.
En segundo lugar, este análisis se complementó identificando el tipo de palabra según su formación (palabra primitiva, derivada, compuesta y parasintética). De igual modo, se determina la clase de palabra a la que pertenece, esto es, sustantivo, adjetivo, verbo, adverbio, preposición, conjunción, artículo, pronombre.

Luego, se determinó el fenómeno de innovación léxica que se pone de manifiesto en la palabra o frase. Así, se determinó si se produce préstamos lingüísticos, calcos, descomposiciones, composiciones, derivaciones, alteraciones del modelo tradicional o creaciones sistemáticas.

Finalmente, se expresó el significado de la palabra según la normativa de la lengua, así como se indicó el equivalente léxico dentro de la variedad estándar del castellano. 


\begin{tabular}{|c|c|c|c|c|c|c|c|c|}
\hline & 苞 & 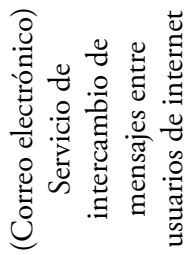 & 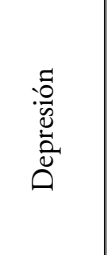 & 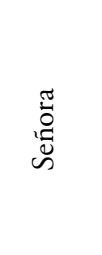 & 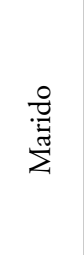 & $\frac{\dot{o}}{\stackrel{0}{U}}$ & 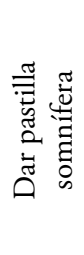 & 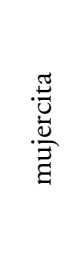 \\
\hline & 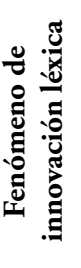 & 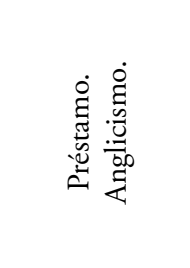 & 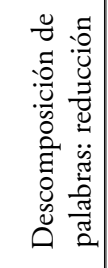 & 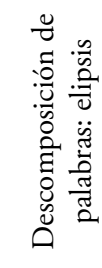 & 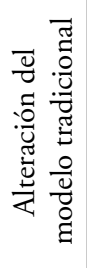 & 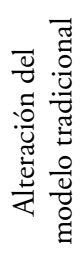 & 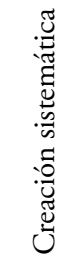 & 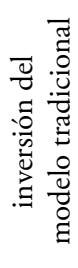 \\
\hline \multicolumn{2}{|c|}{ 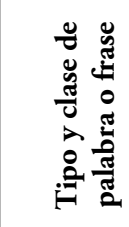 } & 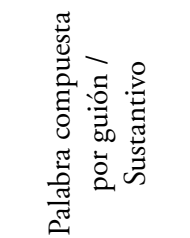 & 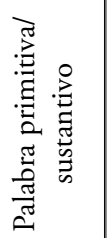 & 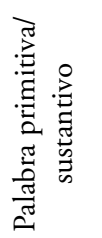 & 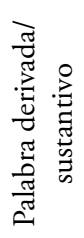 & 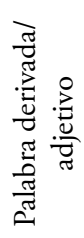 & 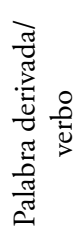 & 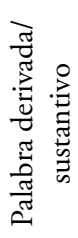 \\
\hline \multirow{7}{*}{ 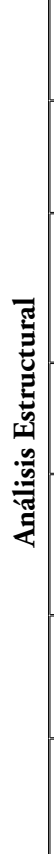 } & $\stackrel{\circ}{\stackrel{5}{5}}$ & & & & : & స్ & $\pi$ &.$\widetilde{ت}$ \\
\hline & 串 & & & & & & & \\
\hline & 苞 & "তี & & & & & & \\
\hline & $\stackrel{\circ}{\stackrel{2}{\Xi}}$ & & & & & & & \\
\hline & 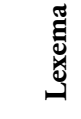 & u & $\frac{\tilde{a}}{\frac{\tilde{\theta}}{0}}$ & 可 & 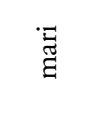 & $\stackrel{\circ}{\mathcal{U}}$ & $\begin{array}{l}\tilde{\partial} \\
\tilde{z}\end{array}$ & 墕 \\
\hline & 串 & & & & & & & \\
\hline & 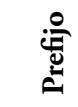 & & & & & & & \\
\hline & & 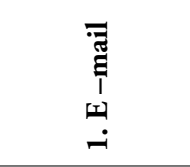 & $\begin{array}{l}\text { ì } \\
\text { ì } \\
\dot{0}\end{array}$ & r & 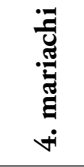 & $\begin{array}{l}\frac{5}{\pi} \\
\frac{\pi}{0} \\
\text { ¿ } \\
\dot{n}\end{array}$ & 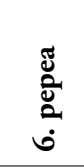 & 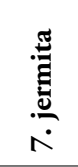 \\
\hline
\end{tabular}




\begin{tabular}{|c|c|c|c|c|c|c|c|c|c|c|}
\hline & 营 & $\begin{array}{l}\text { ठ } \\
\curvearrowleft\end{array}$ & $\frac{8}{2}$ & 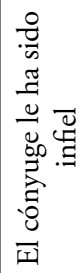 & 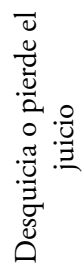 & $\begin{array}{l}\frac{\mathbb{J}}{U} \\
\frac{\tilde{J}}{\tilde{J}}\end{array}$ & 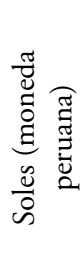 & 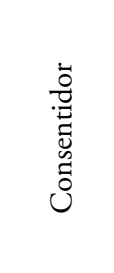 & 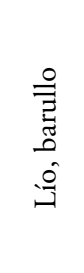 & 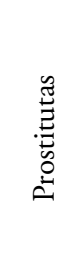 \\
\hline & 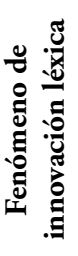 & 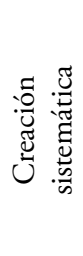 & 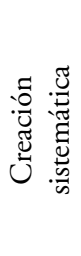 & 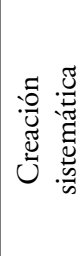 & 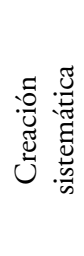 & 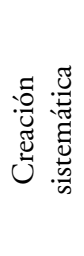 & 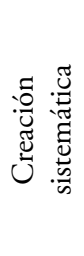 & 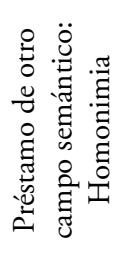 & 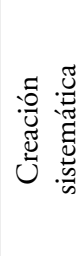 & 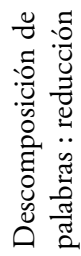 \\
\hline & 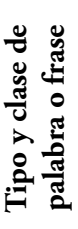 & 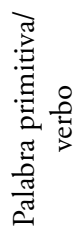 & 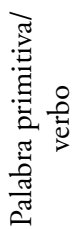 & 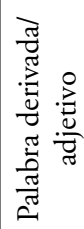 & 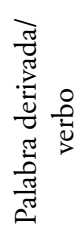 & 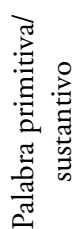 & 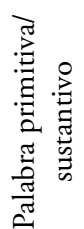 & 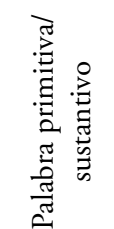 & 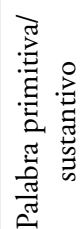 & 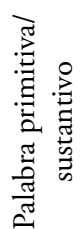 \\
\hline \multirow{8}{*}{ 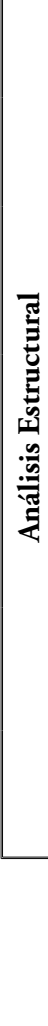 } & $\stackrel{\circ}{\mathscr{S}}$ & & & $\frac{8}{Z}$ & 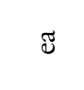 & & & & & \\
\hline & $\stackrel{\circ}{\stackrel{ }{\Xi}}$ & & & & & & & & & \\
\hline & בُّ & & & & & & & & & \\
\hline & 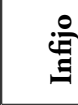 & & & & & & & & & \\
\hline & : & $\begin{array}{l}\text { ․․ } \\
\text { 矛 }\end{array}$ & 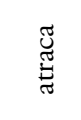 & 胥 & $\vec{\sigma}$ & $\stackrel{\widetilde{E}}{\Xi}$ & 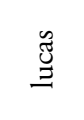 & $\begin{array}{l}\frac{\pi}{\mathbb{\Xi}} \\
\underset{ت}{\tilde{E}}\end{array}$ & $\begin{array}{l}\widetilde{U} \\
0 \\
0\end{array}$ & $\begin{array}{l}\stackrel{n}{0} \\
0 \\
0 \\
0\end{array}$ \\
\hline & $\stackrel{\circ}{\stackrel{0}{S}}$ & & & & & & & & & \\
\hline & 裉 & & & & & & & & & \\
\hline & & 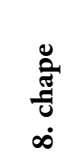 & 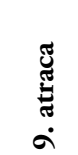 & 월 & $\begin{array}{l}\stackrel{\Xi}{\Xi} \\
\stackrel{\Xi}{\Xi} \\
\stackrel{\Xi}{\beth}\end{array}$ & 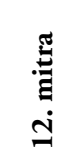 & $\frac{\tilde{Z}}{\underline{E}}$ & 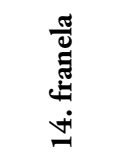 & $\begin{array}{l}\text { ڤே } \\
\dot{0} \\
\dot{0}\end{array}$ & $\begin{array}{l}\stackrel{0}{0} \\
0 \\
0 \\
0 \\
0 \\
0\end{array}$ \\
\hline
\end{tabular}




\begin{tabular}{|c|c|c|c|c|c|c|c|c|c|c|}
\hline & 苞 & 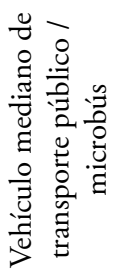 & 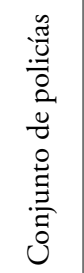 & 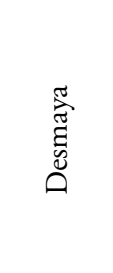 & 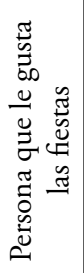 & 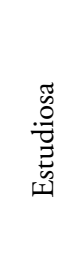 & 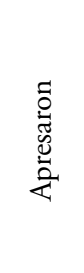 & 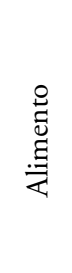 & 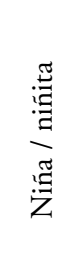 & 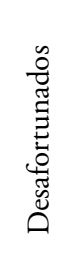 \\
\hline & 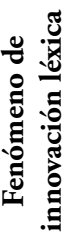 & 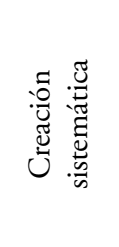 & 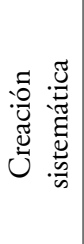 & 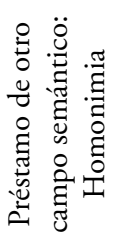 & 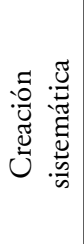 & 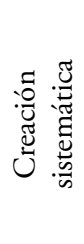 & 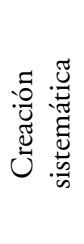 & 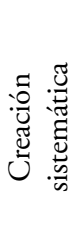 & 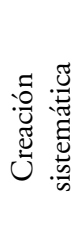 & 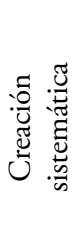 \\
\hline \multicolumn{2}{|c|}{ 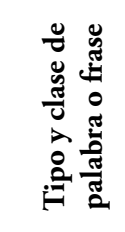 } & 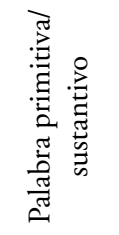 & 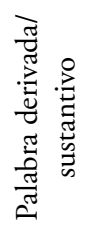 & 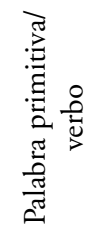 & 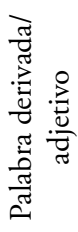 & 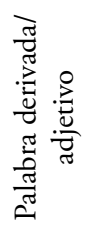 & 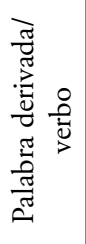 & 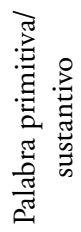 & 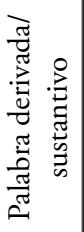 & 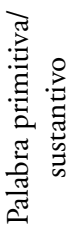 \\
\hline \multirow{7}{*}{ 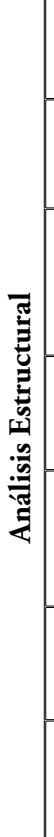 } & $\stackrel{\circ}{5}$ & & 㞼 & & $\stackrel{\circ}{0}$ & ָี & 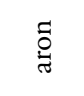 & &.$\widetilde{G}$ & \\
\hline & $\stackrel{\circ}{\stackrel{2}{g}}$ & & & & & & & & & \\
\hline & 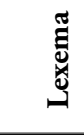 & & & & & & & & & \\
\hline & 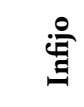 & & & & & & & & & \\
\hline & 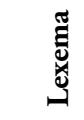 & $\begin{array}{l}\overrightarrow{\vec{E}} \\
\text { है }\end{array}$ & है & $\frac{\tilde{\sigma}}{\mathfrak{\Xi}}$ & કี & $\begin{array}{l}\text { U్ } \\
\text { ]్ }\end{array}$ & 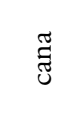 & $\begin{array}{l}\text { \& } \\
\text { हี } \\
0\end{array}$ & 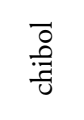 & $\stackrel{\mathscr{\sharp}}{\overparen{\Xi}}$ \\
\hline & $\stackrel{\circ}{\stackrel{0}{g}}$ & & & & & & & & & \\
\hline & 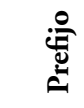 & & & & & & & & & \\
\hline & & 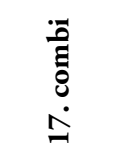 & 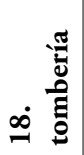 & 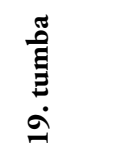 & $\begin{array}{l}\stackrel{0}{0} \\
\stackrel{0}{0} \\
\dot{d}\end{array}$ & 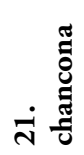 & ¿் & $\begin{array}{l}\stackrel{8}{8} \\
\dot{\Xi} \\
\dot{d} \\
\dot{\sim}\end{array}$ & せ্ّ & 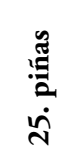 \\
\hline
\end{tabular}


ESQUEMA DE ANÁLISIS SÉMICO EN UNA PALABRA

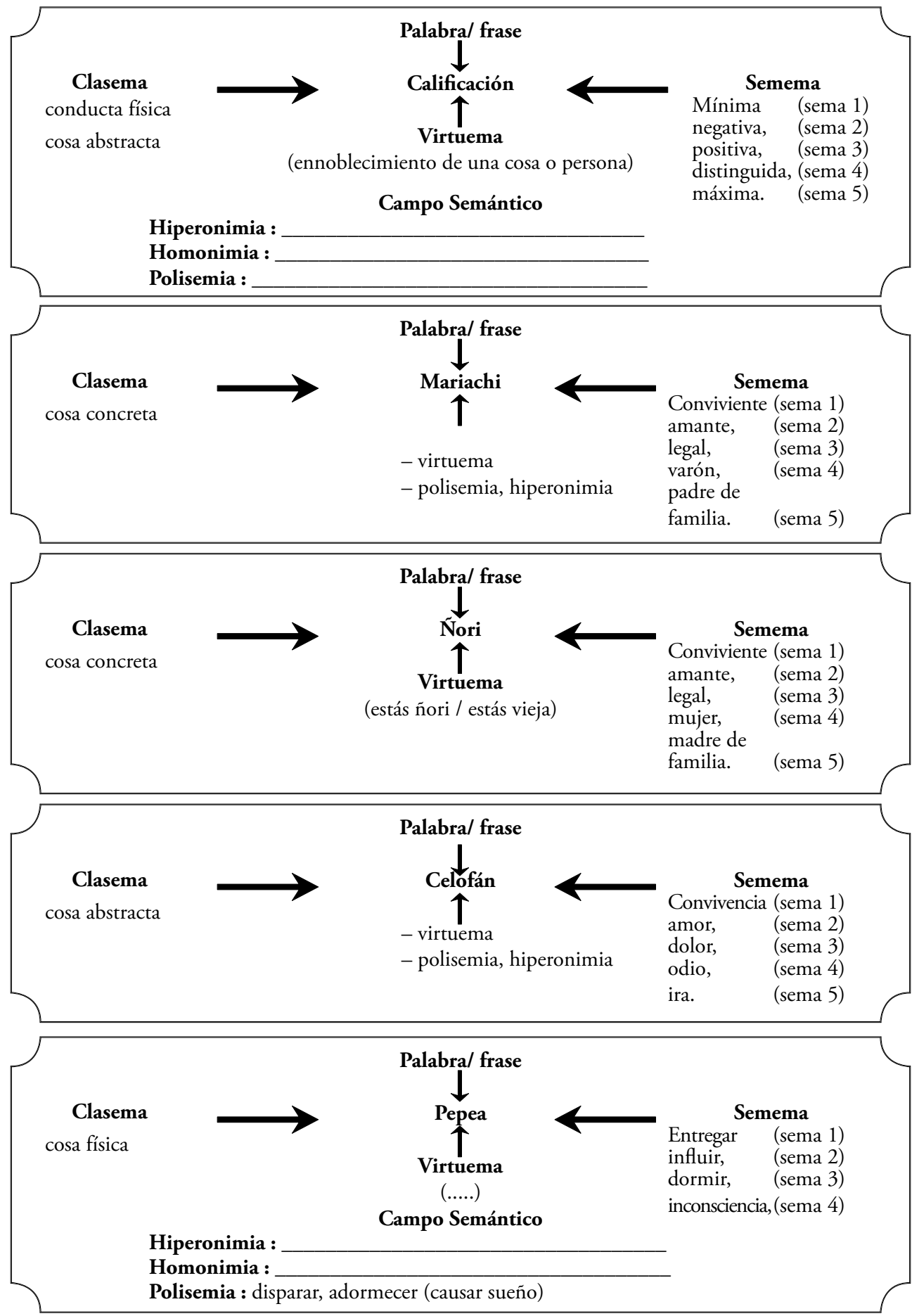




\section{Resultados}

A la luz de los resultados del análisis lexicológico podemos interpretar que el principal procedimiento de innovación léxica es la creación sistemática; en segundo orden, el préstamo de lexemas de otro campo semántico (homonimia), que forman parte de la variedad estándar y normativa de la lengua. En tercer lugar, se encuentra el procedimiento de innovación léxica denominado alteración del modelo tradicional en la modalidad de derivación léxica. Por tanto, el tipo de palabra que se prefiere en la estructuración léxica es la primitiva y de clase sustantivo. En este sentido, se prefieren palabras de un solo lexema.

Frente a los resultados del análisis semántico podemos inferir que el principal sema empleado - que se constituye, por tanto, en un archisemema - es el de fuerza; pues, en la mayoría de palabras analizadas se encuentra este rasgo mínimo de significado.

Igualmente, dentro de los clasemas que son recurrentes se encuentran los de conducta física y cosa concreta. Así, no se presentan los clasemas de cosa abstracta, conducta abstracta. Los virtuemas no son muy empleados como rasgos mínimos de significación en las distintas innovaciones semánticas.

\section{Conclusiones}

La primera conclusión a inferir de este estudio lingüístico descriptivo es que los cambios en la lengua están directamente relacionados con las necesidades de los hablantes en torno a una mayor comprensión y objetivación del mundo en que es- tán insertos. Por tanto, estos cambios se dan de acuerdo a la realidad sociohistórica y geográfica de los individuos, por ser el lenguaje un fenómeno social.

De este modo, se dedujo que las variaciones lingüísticas en el plano léxico-semántico surgen de diversa manera con la finalidad de que las personas comprendan y dominen su entorno.

Asimismo, se registró un total de 100 palabras, demostrando que periodismo y lenguaje están estrechamente unidos, debido a que refleja los cambios de la lengua del público a que están dirigidos sus mensajes y también tiene incidencia en la aceptación masiva de estas innovaciones.

Por otra parte, se infiere que la prensa escrita 'amarilla' también contribuye a que la lengua subestándar ingrese progresivamente al lenguaje del ciudadano común, lo que se evidencia con la aceptación y comprensión por parte de sus receptores de conceptos tales como, teclito, pelona, choborra, pezziduri, entre muchos otros, contribuyendo de esta manera a la difusión de parte del conocimiento sociolingüístico peruano.

Las características del repertorio léxicosemántico de la prensa amarilla en Lima Metropolitana está dada por las innovaciones encontradas y sistematizas según las categorías existentes en la lingüística, las cuales corresponden a:

La creación sistemática por neologismos, debido a que la mayoría de ellas no mantiene su estructura original (morfológica) y su significado (semántica). No se 
emplean para ello voces griegas y latinas como lo suelen efectuar en situaciones de comunicación congruentes con la variedad estándar.

Se registró un número significativo de sustantivaciones, utilizando nuevos lexemas $\mathrm{y}$, los distintos afijos y sufijos con el fin de dar distintas denotaciones a los conceptos y mensajes emitidos.

La última conclusión es que los principales factores que influyen en las innovaciones léxicas recopiladas en el corpus de estudio estuvieron dadas mayoritariamente por:

a) Neologismos por creación sistemática de palabras (monses, bamba, maricatú, kechi)

b) Préstamos lingüísticos, principalmente castellanos (fichita, tía, tercia), pero dándole otro significado, generando la homonimia que hoy forman parte del lenguaje cotidiano subestándar de Lima Metropolitana.

c) Alteración del modelo tradicional, a través del procedimiento de la derivación (boloñas, barrunto, jaraneo).

\section{Nota}

1. Es la parte de la gramática que describe las reglas por las que las unidades significativas se combinan en oraciones.

\section{Referencias bibliográficas}

AKMAJIAN, Adrián; DEMERS, Richard A; HARMISH, Robert M. Lingüística: una introducción al lenguaje y la comunicación. Madrid, Alianza, 1995.
BRIZ, A. El español coloquial: Situación y uso. Madrid, Arco/Libros, 1996.

COSERIU, Eugenio. Sincronía, diacronía e historia. El problema del cambio lingüístico. Madrid, Gredos, 1958.

COSERIU, Eugenio. Teoría del lenguaje y lingüística general. Madrid, Gredos, 1967.

FINEGAN, E., \& BIBER, D. Register and social dialect variation: An integrated approach. En: D. Biber \& E. Finegan (Eds.), Sociolinguistic perpectives on register. Nueva York: Oxford Univesity, 1994. 315-347 pp.

GUMPERZ, John; BENNETT, Adrian.

Lenguaje y cultura. Madrid, Anagrama, 1981.

HAENSCH, G. Aspectos prácticos de la elaboración de diccionarios. En: G. Haensch, L. Wolf, S Ettinger, \& R. Werner. La lexicografía: De la lingüística teórica a la lexicografía práctica. Madrid, Gredos, 1982a. 395-534 pp.

HODGE, R. \& KRESS, G. Social semiotics, style and ideology. En: N. Coupland \& A. Jaworski (Eds.), Sociolinguistics: A reader and coursebook. Chippenham, Inglaterra: Palgrave, 1997. 49 -54 pp.

HOLMES, J. An introduction to sociolinguistics . Harlow, England: Pearson Educational. 2001.

IRVINE, J. T. Formality and informality in communicative events. En: J. Baugh \& J. Sherzer (Eds.), Language in use: Reading in sociolinguistics. Englewood Cliffs, Nueva Jersey: Prentice-Hall, 1984. $211-228$ pp.

LANGER, John. La televisión sensacionalista. Periodismo popular y las otras noticias. Barcelona, Paidos, 2000. 213 p. 
LABOV, W. Modelos sociolingüísticos. Trad. J. M. Marinas Herreras. Madrid, Cátedra, 1983.

LABOV, W. Field methods of the project on linguistic change and variation. En: J. Baugh \& J. Sherzer (Eds.), Language in use: Reading in sociolinguistics . Englewood Cliffs, Nueva Jersey, Prentice-Hall, 1984. 28-53 pp.

LEWANDOWSKI, Theodor. Diccionario de lingüística. España, Cátedra, 2000.

LUQUE, J. D.; PAMIES, A.; \& MANJÓN, F. J. El diccionario del insulto. Barcelona, Península, 2000.

LOMAS, Carlos. Cómo enseñar a hacer cosas con las palabras. Vol. II. Barcelona, Paidos, 1999.

MATA, María Cristina. Interrogaciones sobre el público. Universidad Nacional de Córdoba. Mimeo, 2000. 19 p.

MATA NAVARRETE, N. Léxico agrícola de San José de Ramos. Tesis de licenciatura no publicada, UNAM. México, 1985.
MALMBERG, Bertil. La lengua el hombre. Madrid, ISTMO, 1966.

MOUIN, Georges. Diccionario de Lingüística. París, Labor, 1979.

REALACADEMIAESPAÑOLA. Diccionario de la lengua española. 22a ed. Madrid, Espasa Calpe, 2001.

RODRÍGUEZ, Francisco. Lingüística estructural. Madrid, Gredos, 1969.

RODRÍGUEZ, Gustavo. Cuaderno de filología, 12. Antofagasta, Universidad de Chile, 1980.

RODRÍGUEZ, Gustavo. Status del español en el periodismo de hoy. Revista de la Sociedad Argentina de Lingüística, Año 2, $\mathrm{N}^{\circ}$ 2. Universidad de Tucumán, Argentina, 1994. $27-40$ pp.

SAPERAS, Eric. Los efectos cognitivos de la comunicación de masas. España, Ariel S.A., 1987.

SCHAMM, Wilbur. La ciencia de los humanos. España, Paraninfo, 1992. 